\title{
Towards 3D Understanding of Non-spherical Nanoparticles by Transmission Kikuchi Diffraction (TKD) for Improved Particle Size Distribution by Electron Microscopy
}

Vasile-Dan Hodoroaba ${ }^{1}$, Grzegorz Cios ${ }^{2}$, Tomasz Tokarski ${ }^{2}$, Ulrich Mansfeld ${ }^{1}$, Erik Ortel ${ }^{1}$, Johannes Mielke $^{1}$, Francesco Pellegrino ${ }^{3}$ and Valter Maurino ${ }^{3}$

${ }^{1}$ Federal Institute for Materials Research and Testing (BAM), Berlin, Berlin, Germany, ${ }^{2}$ AGH University of Science and Technology, Krakow, Malopolskie, Poland, ${ }^{3}$ Università di Torino, Turin, Piemonte, Italy

Accurate measurement of nanoparticle (NP) size for particles with non-spherical shape constitutes a challenging analytical problem in principle for all methods able to probe the size of nanoparticles [1]. Various versions of electron microscopy produce conventionally 2D projection images which may induce significant errors in the evaluation of the true, 3D size and, hence, are not suited for the metrological qualification of size measurement of nanoparticles of more complex shape. Electron tomography (ET) by sequential tilting the TEM sample (holder) is one extensive approach able to address the 3D shape of NPs by reconstruction of $3 \mathrm{D}$ images from $2 \mathrm{D}$ projection images [2-4]. Another way of extracting the accurate $3 \mathrm{D}$ size of crystalline nanoparticles is high-resolution TEM coupled with reconstruction from SAED on applied on single NPs [5]. This method is also time-consuming and is impractical to be applied on a statistically relevant number of NPs. Further approaches like manual identification of the well-defined shape of the NPs from high-resolution SEM micrographs of type SE-InLens showing a superior topography contrast might also reveal virtual 3D morphology, see Figure 1a.

In this paper one ingenious approach is applied to determine the exact orientation of bipyramidal $\mathrm{TiO}_{2}$ NPs prepared with good dispersion as almost isolated particles on a carbon TEM grid. The advantages of the recently developed high-throughput Transmission Kikuchi Diffraction (TKD) are used to identify quickly and reliably the orientation of the crystalline $\mathrm{TiO}_{2}$ NPs bipyramids (anatase) on a statistically relevant number of particles [5]. The prerequisite is the successful preparation of the anatase NPs as (mostly) single, non-overlapping particles (see Figure 1b) on an electron transparent substrate, i.e. here carbon foil on typical TEM grids [6].

Figure $1 \mathrm{~b}$ shows a representative inverse pole figure (IPF) map overlaid with the so-called image quality map (with bright spots meaning good signal) as extracted from the TKD signals obtained from $\mathrm{TiO}_{2}$ bipyramidal NPs prepared with good dispersion from liquid suspension on a carbon TEM grid. Note the good quality of the TKD signal generated by the anatase NPs (Figure 1c).

From the analysis of the experimental pole figures (Figure 2a) one can conclude that the bipyramids do not have one single orientation. Simulations of the pole figures overlaid on experimental pole figure (coloured accordingly to IPF in Figure $1 b$ ) for anatase bipyramids in Figure $2 b$ and $c$ indicate that the particles rather lie on the substrate, with their long axis parallel to the substrate ('balancing' at the place of their thickest region). Considerable part of the NPs lies on the $\{101\}$ facets (Figure 2c). Due to touching particles (mechanical support of surrounding NPs), particle orientations far from lying on $\{101\}$ facets or balancing along long axis can be also found in the investigated sample. In the next steps, the NP size measurement uncertainties associated to the various orientations of the anatase NPs as prepared and analysed here by electron microscopy will be quantified [7]. 
a) SEM

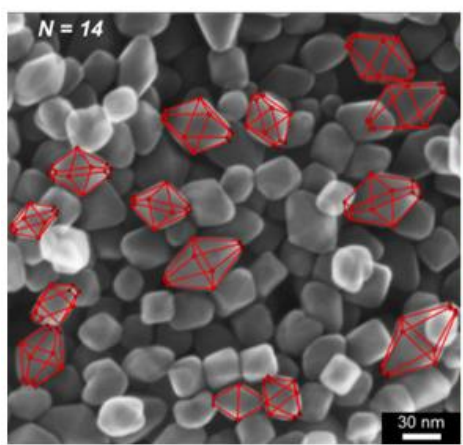

b) IPF-Z + IQ

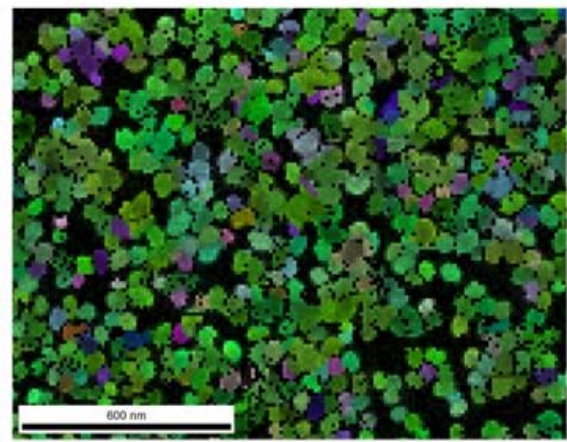

c) TKD pattern

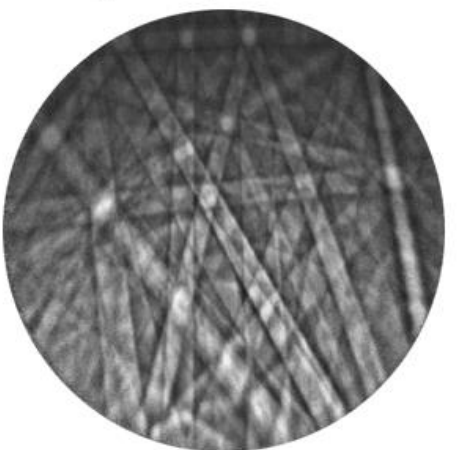

Figure 1. Figure 1. a) High resolution SEM image (SE InLens detector) providing high topography contrast suited to estimate the 3D orientation of TiO2 NPs of bipyramidal shape, b) Inverse pole figure (IPF) map overlaid with the so-called image quality map (with bright spots meaning good signal) as extracted from the TKD signals of TiO2 bipyramidal NPs prepared by drop casting the particles on a carbon foil TEM grid with minimum overlap, and c) anatase TKD pattern (just to illustrate the available pattern quality).

a) Experimental pole figures

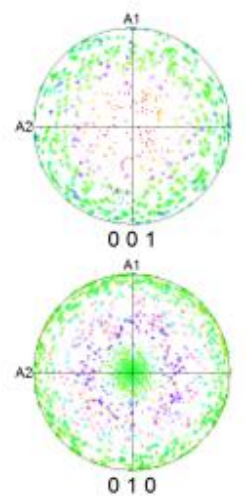

b) Overlaid theoretical pole figures for balancing NPs (red line)
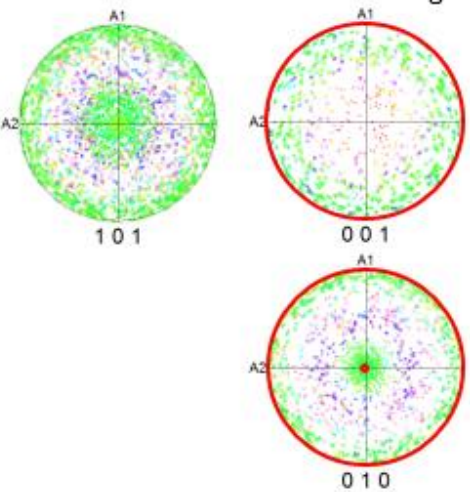

c) Overlaid theoretical pole figures for NPs lying on 101 facets (red line)
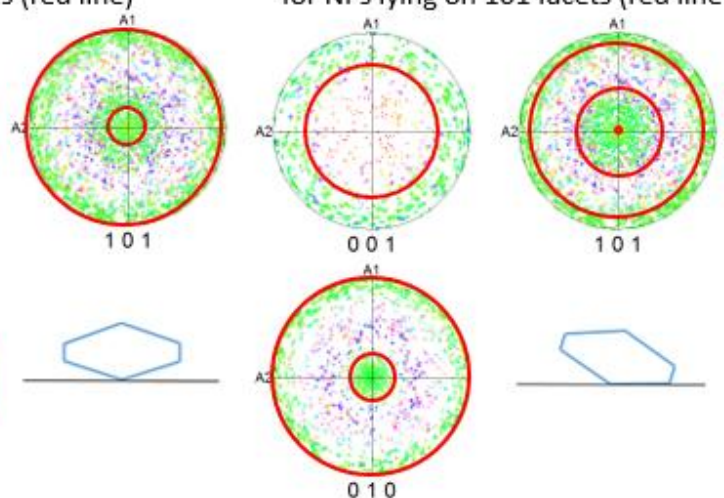

Figure 2. a) Experimental pole figures corresponding to TiO2 bipyramidal NPs as in Figure 1, b) overlaid theoretical pole figures in case of 'balancing' NPs, and c) overlaid theoretical pole figures in case of NPs lying on $\{101\}$ facets.

\section{References}

[1] F Babick et al, J. Nanopart. Res. 18:158 (2016), p. 1.

[2] E A F Van Doren et al, Journal of Nanobiotechnology (2011), 9:17.

[3] E Padgett et al, Microsc. Microanal. 23 (2017), p. 1150.

[4] M Hayashida, M Malac, Meas. Sci. Technol. 28 (2017), 087001.

[5] N Wollschläger et al, Mater. Charact. 131 (2017), p. 39.

[6] U Mansfeld et al, Microsc. Microanal. 25 (2019), p. 2328.

[7] This project has received funding from the EMPIR programme co-financed by the Participating States and from the European Union's Horizon 2020 research and innovation Programme. 